\title{
A comparison of publication to TBI burden ratio of low- and middle-income countries versus high-income countries: how can we improve worldwide care of TBI?
}

\author{
Maria Pia Tropeano, MD, ${ }^{1}$ Riccardo Spaggiari, MD, ${ }^{1}$ Hernán lleyassoff, ${ }^{1}$ Kee B. Park, MD, MPH, ${ }^{2}$ \\ Angelos G. Kolias, MD, PhD,, Peter J. Hutchinson, MBBS, PhD, FMedSci, ${ }^{3,4}$ and \\ Franco Servadei, MD'
}

\begin{abstract}
${ }^{1}$ Department of Neurosurgery, Humanitas Clinical and Research Hospital and Humanitas University, Milano, Italy; ${ }^{2}$ Global Neurosurgery Initiative, Program in Global Surgery and Social Change, Department of Global Health and Social Medicine, Harvard Medical School, Boston, Massachusetts; ${ }^{3}$ National Institute for Health Research (NIHR) Global Health Research Group on Neurotrauma, University of Cambridge; and 'Division of Neurosurgery, Department of Clinical Neurosciences, Addenbrooke's Hospital and University of Cambridge, United Kingdom
\end{abstract}

OBJECTIVE Traumatic brain injury (TBI) is a global public health problem and more than $70 \%$ of trauma-related deaths are estimated to occur in low- and middle-income countries (LMICs). Nevertheless, there is a consistent lack of data from these countries. The aim of this work is to estimate the capacity of different and heterogeneous areas of the world to report and publish data on TBI. In addition, we wanted to estimate the countries with the highest and lowest number of publications when taking into account the relative TBI burden.

METHODS First, a bibliometric analysis of all the publications about TBI available in the PubMed database from January 1,2008 , to December 31,2018 , was performed. These data were tabulated by country and grouped according to each geographical region as indicated by the WHO: African Region (AFR), Region of the Americas (PAH), South-East Asia Region (SEAR), European Region (EUR), Eastern Mediterranean Region (EMR), and Western Pacific Region (WPR). In this analysis, PAH was further subdivided into Latin America (AMR-L) and North America (AMR-US/Can). Then a "publication to TBI volume ratio" was derived to estimate the research interest in TBI with respect to the frequency of this pathology.

RESULTS Between 2008 and 2018 a total of 8144 articles were published and indexed in the PubMed database about TBI. Leading WHO regions in terms of contributions were AMR-US/Can with 4183 articles (51.36\%), followed by EUR with 2003 articles (24.60\%), WPR with 1507 (18.50\%), AMR-L with 141 articles (1.73\%), EMR with 135 (1.66\%), AFR with 91 articles (1.12\%), and SEAR with 84 articles (1.03\%). The highest publication to TBI volume ratios were found for AMR-US/Can (90.93) and EUR (21.54), followed by WPR (8.71) and AMR-L (2.43). Almost 90 times lower than the ratio of AMR-US/Can were the ratios for AFR (1.15) and SEAR (0.46).

CONCLUSIONS An important disparity currently exists between countries with a high burden of TBI and those in which most of the research is conducted. A call for improvement of data collection and research outputs along with an increase in international collaboration could quantitatively and qualitatively improve the ability of LMICs to ameliorate TBI care and develop clinical practice guidelines.

https://thejns.org/doi/abs/10.3171/2019.8.FOCUS19507

KEYWORDS global neurosurgery; traumatic brain injury; publication; LMICs; research

$\mathrm{T}$ RAUMATIC brain injury (TBI) is a major public health problem globally, as it is the most severe injury type among trauma-related accidents ${ }^{22}$ and therefore affects approximately 69 million people every year. ${ }^{10}$ Epidemiological data from different geographical regions and countries are hampered by several factors. For example, mild TBI cases, which usually comprise $80 \%-90 \%$ of all TBI cases, are often not reported or are underreported because very few of them require hospitalization. ${ }^{5}$ Furthermore, there is a lack of consistent data capture due

ABBREVIATIONS AFR = African Region; AMR-L = Latin America; AMR-US/Can = North America; EMR = Eastern Mediterranean Region; EUR = European Region; HIC = high-income country; ICP = intracranial pressure; LMICs = low- and middle-income countries; PAH = Region of the Americas; SEAR = South-East Asia Region; TBI = traumatic brain injury; WPR = Western Pacific Region.

SUBMITTED June 28, 2019. ACCEPTED August 20, 2019.

INCLUDE WHEN CITING DOI: 10.3171/2019.8.FOCUS19507. 
TABLE 1. Publication output according to region, along with main contributor country

\begin{tabular}{|c|c|c|c|c|c|}
\hline \multirow[b]{2}{*}{ Region } & \multicolumn{3}{|c|}{ No. of Publications } & \multirow[b]{2}{*}{ Main Contributor } & \multirow[b]{2}{*}{ No. of Publications } \\
\hline & 10 Yrs (\%) & Average & 2018 Only (\%) & & \\
\hline AMR-US/Can & $4183(51.36)$ & 418.3 & 759 (41.91) & US & 3427 \\
\hline EUR & $2003(24.60)$ & 200.3 & $507(28.00)$ & United Kingdom & 451 \\
\hline WPR & $1507(18.50)$ & 150.7 & $392(21.65)$ & Australia & 532 \\
\hline AMR-L & $141(1.73)$ & 14.1 & $56(3.09)$ & Brazil & 50 \\
\hline EMR & $135(1.66)$ & 13.5 & $47(2.60)$ & Iran & 47 \\
\hline AFR & 91 (1.12) & 9.1 & $25(1.38)$ & South Africa & 57 \\
\hline SEAR & $84(1.03)$ & 8.4 & $25(1.38)$ & India & 66 \\
\hline Total & 8144 & 814.4 & 1811 & & \\
\hline
\end{tabular}

The average value was obtained by dividing the number of publications (productivity) over 10 years by the number 10. The "main contributor" is the country with the most publications in that region along with its respective number of publications.

to poor injury surveillance/reporting systems in many low- and middle-income countries (LMICs) but also in some high-income countries (HICs). ${ }^{21}$ LMICs face the greatest burden of trauma, as more than $70 \%$ of traumarelated deaths are estimated to occur there. ${ }^{10}$ Differences between LMICs and HICs exist in terms of infrastructure, resources, availability of staff, ${ }^{24}$ and epidemiology, as patients tend to be younger and road traffic accidents are the most prevalent injury mechanism in LMICs..$^{15}$ Because of these discrepancies, the development of relevant contextspecific clinical practice guidelines useful for LMICs requires the publication of research and other TBI-related papers from these countries. Previous bibliometric studies have already highlighted the contribution of different countries in the worldwide neurosurgical literature, $, 2,16,25$ but none of them have taken into account the respective incidence of disease for each region. We conducted a bibliometric analysis of TBI-related papers published in the last 10 years and in 2018, and after collecting volumes of the pathology for each geographical region, we estimated the capacity of different and heterogeneous areas of the world to report and publish data on TBI. In addition, we wanted to estimate countries with the highest and lowest number of publications when taking into account the relative TBI burden.

\section{Methods}

\section{Publication Capacity Across WHO Regions}

We conducted a bibliometric analysis of all the publications available in the PubMed database from January 1, 2008, to December 31, 2018. Related publications were identified by searching for the MeSH term "TBI traumatic brain injury." As previously described in the literature, ${ }^{23}$ we used the Boolean operators (AND, OR, and NOT) in our search string to collect the number of publications for each country. For example, to obtain TBI-related publications in Italy, we searched for "(TBI traumatic brain injury[MeSH Terms]) AND Italy." Moreover, an additional search was conducted with the publication date filter set only to the year 2018. The data were then tabulated for country and grouped according to each geographical region as indicated by the WHO (https://www.who. int/choice/demography/by_country/en/): African Region (AFR), Region of the Americas (PAH), South-East Asia Region (SEAR), European Region (EUR), Eastern Mediterranean Region (EMR), and Western Pacific Region (WPR). PAH was further subdivided as previously described by Dewan et al..$^{10}$ into North America (AMR-US/ Can) and Latin America (AMR-L).

\section{Publication Ratio}

In the second part of our study we derived a "publication ratio" for each WHO region by dividing the average number of published articles in that region (numerator, obtained by dividing the documents produced in 10 years by the number 10) by the volume of TBI in the population inhabiting that region (denominator). A similar publication ratio has already been described in the literature by AlShahi et al. ${ }^{2}$ As previously performed by Corley et al., ${ }^{9}$ the volume of TBI was obtained from the estimation conducted by Dewan et al. ${ }^{10}$ (number of million new TBI cases/ year). We subdivided the PAH into North America (AMRUS/Can) and Latin America (AMR-L) to have comparable data. Data were analyzed using Microsoft Excel.

\section{Results}

\section{Publication Output Worldwide}

Between 2008 and 2018 a total of 8144 articles were published and indexed in the PubMed database about TBI. Leading WHO regions in terms of contributions were AMR-US/Can with 4183 articles (51.36\%), followed by EUR with 2003 articles (24.60\%), WPR with 1507 (18.50\%), AMR-L with 141 articles (1.73\%), EMR with 135 (1.66\%), AFR with 91 articles (1.12\%), and SEAR with 84 articles (1.03\%; Table 1). These percentages are represented graphically in Fig. 1. The main contributors in each WHO region were the US for AMR-US/ CAN (81.9\% of publications from this region), the United Kingdom for EUR (22.52\% of publications), Australia for WPR (35.30\%), Brazil for AMR-L (35.46\%), Iran for EMR (34.82\%), South Africa for AFR (62.64\%), and India for SEAR (78.57\%). Additionally, in Table 1 we report the number of published papers only for the year 2018 . Comparing 2018 with the entire decade, one can see that 


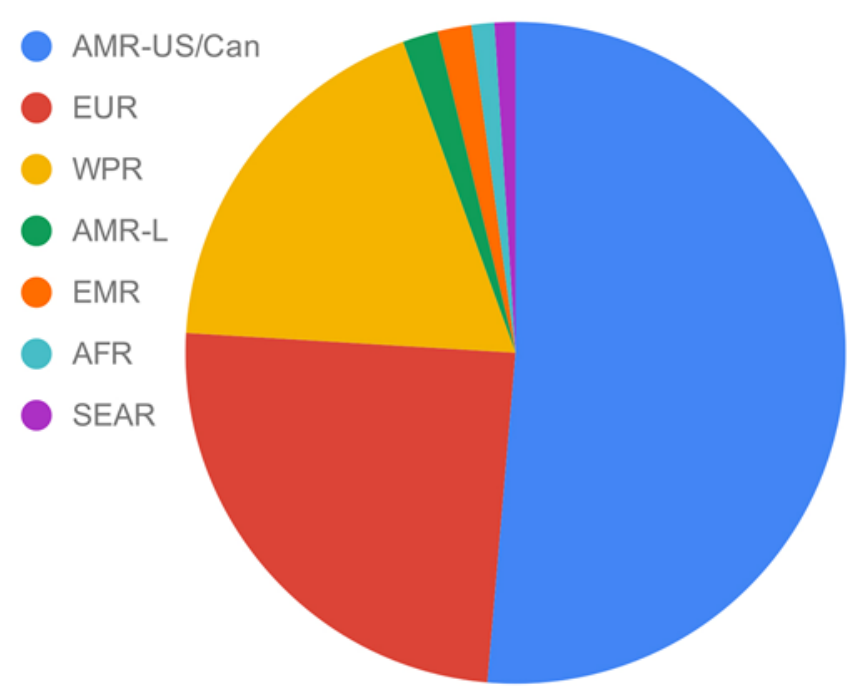

FIG. 1. Proportional contribution of TBI publications over a 10 -year period for each geographic region.

there is a decrease in papers from AMR-US/Can and an increase in papers from all other areas. We subsequently divided all countries into 4 groups according to the number of publications: $>500$ articles, $100-500$ articles, 50100 , and $10-50$. For the papers published in 2018 we used a different scale: $>100$ papers, $50-100,10-50,5-10$, and 1-5 papers (Table 2).

\section{Publication to TBI Volume Ratio}

By dividing the average number of publications per year by the volume of new cases of TBI indicated in million cases per year we obtained a "publication to TBI volume ratio" highlighting a huge heterogeneity among regions. The highest ratios were found for AMR-US/Can (90.93) and EUR (21.54), followed by WPR (8.71) and AMR-L (2.43). Almost 90 times lower than the ratio of the AMRUS/Can region were the ratios for AFR (1.15) and SEAR (0.46; Table 3). This disparity can be appreciated in Fig. 2 , showing the different contributions in percentages in terms of worldwide publication productivity and of TBI cases for each region, and in Fig. 3, reporting the variation of the publication to TBI volume ratio across the globe.

\section{Discussion}

The aim of our study was to compare the worldwide volume of TBI with the published papers on TBI as reported by PubMed. We tabulated all clinical papers published from 2008 to 2018 addressing TBI, subdivided according to the country of origin. In the literature, several articles described the burden of TBI at a continental level. Overall, 295 per 100,000 people per year are affected worldwide by brain trauma according to a meta-analysis by Nguyen et al. ${ }^{19}$ This burden is, however, heterogeneously distributed across the continents. In fact, the incidence of TBI varies from higher numbers in Europe and the US (287.2/100,000 hospital admissions/year in Europe ${ }^{18}$ and 787.1/100,000 emergency department visits/year in the $\mathrm{US}^{27}$ ) to lower numbers in the Middle East Region
TABLE 2. Results by country

\begin{tabular}{cc}
\hline $\begin{array}{c}\text { No. of } \\
\text { Publications }\end{array}$ & \multicolumn{1}{c}{ Countries } \\
\hline Overall & \\
\hline$>500$ & US, Canada, Australia, China \\
\hline $100-500$ & $\begin{array}{c}\text { United Kingdom, Germany, Italy, Sweden, Netherlands, } \\
\text { France, New Zealand, Japan }\end{array}$ \\
\hline $50-100$ & $\begin{array}{c}\text { Switzerland, Israel, South Korea, Taiwan, Ireland, Bel- } \\
\text { gium, Spain, India, South Africa, Finland, Denmark, } \\
\\
\text { Russia, Norway, Brazil }\end{array}$ \\
\hline $10-50$ & $\begin{array}{c}\text { Iran, Poland, Singapore, Austria, Jordan, Argentina, } \\
\text { Turkey, Greece, Colombia, Saudi Arabia, Chile, Hun- }\end{array}$ \\
\hline gary, Portugal, Qatar, Mexico, Lebanon, Malaysia, \\
Pakistan
\end{tabular}

Countries grouped according to their publication output for the 10-year period and 2018 only, indicated as ranges of the number of publications.

(45/100,000 hospital admissions). ${ }^{11}$ Furthermore, in Europe patients are older (with a mean age around 50 years) and the most common mechanism of injury is falls.,21 The volume of TBI is described as specifically afflicting LMICs,$^{15}$ but the literature from these areas reports mostly data from single-center studies. For example, a study by

TABLE 3. Publication to volume ratio by region

\begin{tabular}{lccc}
\hline \multicolumn{1}{c}{ Region } & $\begin{array}{c}\text { TBI Volume } \\
\text { (million cases/yr) }\end{array}$ & $\begin{array}{c}\text { Mean No. of } \\
\text { Publications/Yr }\end{array}$ & Ratio \\
\hline AMR-US/Can & 4.6 & 418.3 & 90.93 \\
\hline EUR & 9.3 & 200.3 & 21.54 \\
\hline WPR & 17.3 & 150.7 & 8.71 \\
\hline AMR-L & 5.8 & 14.1 & 2.43 \\
\hline EMR & 5.8 & 13.5 & 2.33 \\
\hline AFR & 7.9 & 9.1 & 1.15 \\
\hline SEAR & 18.3 & 8.4 & 0.46 \\
\hline
\end{tabular}

${ }^{*}$ TBI volume for each region as reported by Dewan et al. ${ }^{10}$ 


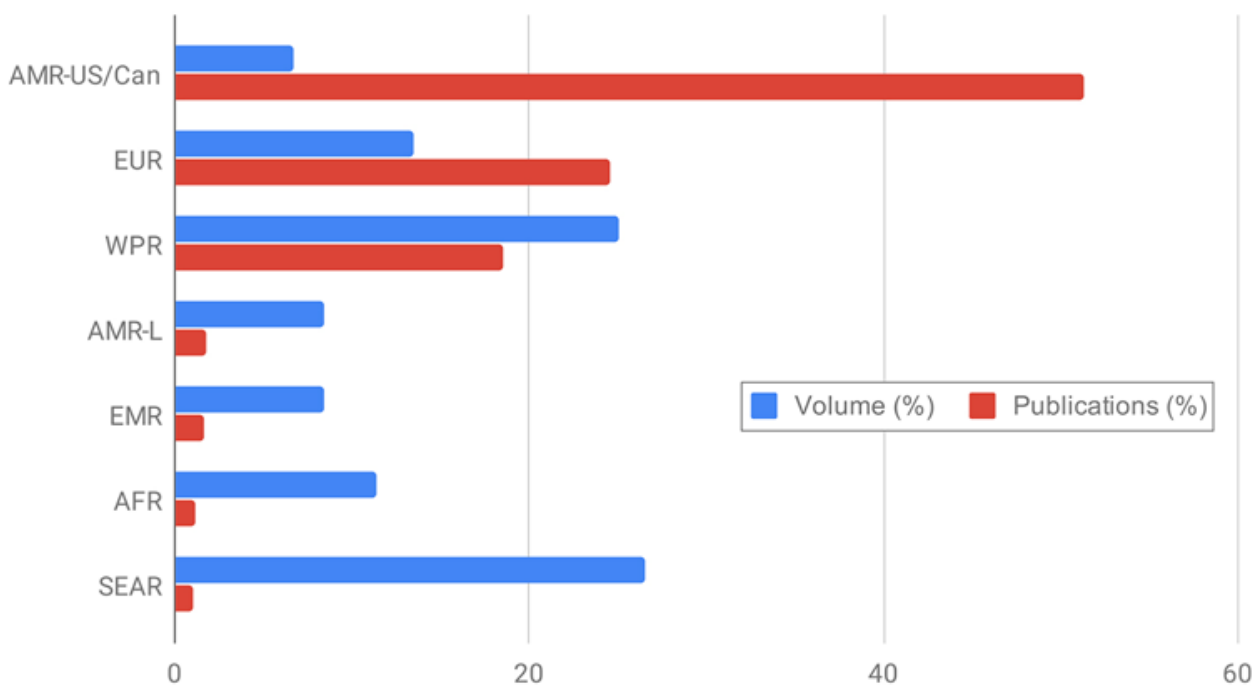

FIG. 2. Comparison of the percentages of total publications and TBI case volumes for each geographic region.

Peeters et al. ${ }^{20}$ described 1200 admitted patients with TBI in a major government hospital in Cambodia. A study by Tran et al..$^{28}$ reported an incidence of 89/100,000 admitted cases/year of severe TBI that was deduced from a retrospective collection of patients with GCS scores $<8$ admitted to a single national referral hospital in Uganda. To have reliable and comparable data across regions in our study, we decided to utilize the volume of cases reported in the article by Dewan et al., ${ }^{10}$ as previously performed in the literature with other objectives. ${ }^{9}$ In fact, Dewan et al..$^{10}$ es- timated the incidence through a sequence of mathematical steps with data obtained through a systematic review of the literature. Using a methodology previously described in the literature, ${ }^{2}$ the aim of our bibliometric analysis was to relate the epidemiology of a WHO region to the number of published papers on TBI in order to form an estimate of the publishing capacity of each region. As detailed in Table 1 and Fig. 1, the largest number of publications were from US/Canada, Europe, and the Western Pacific Region, with Africa and Southeast Asia together responsible for

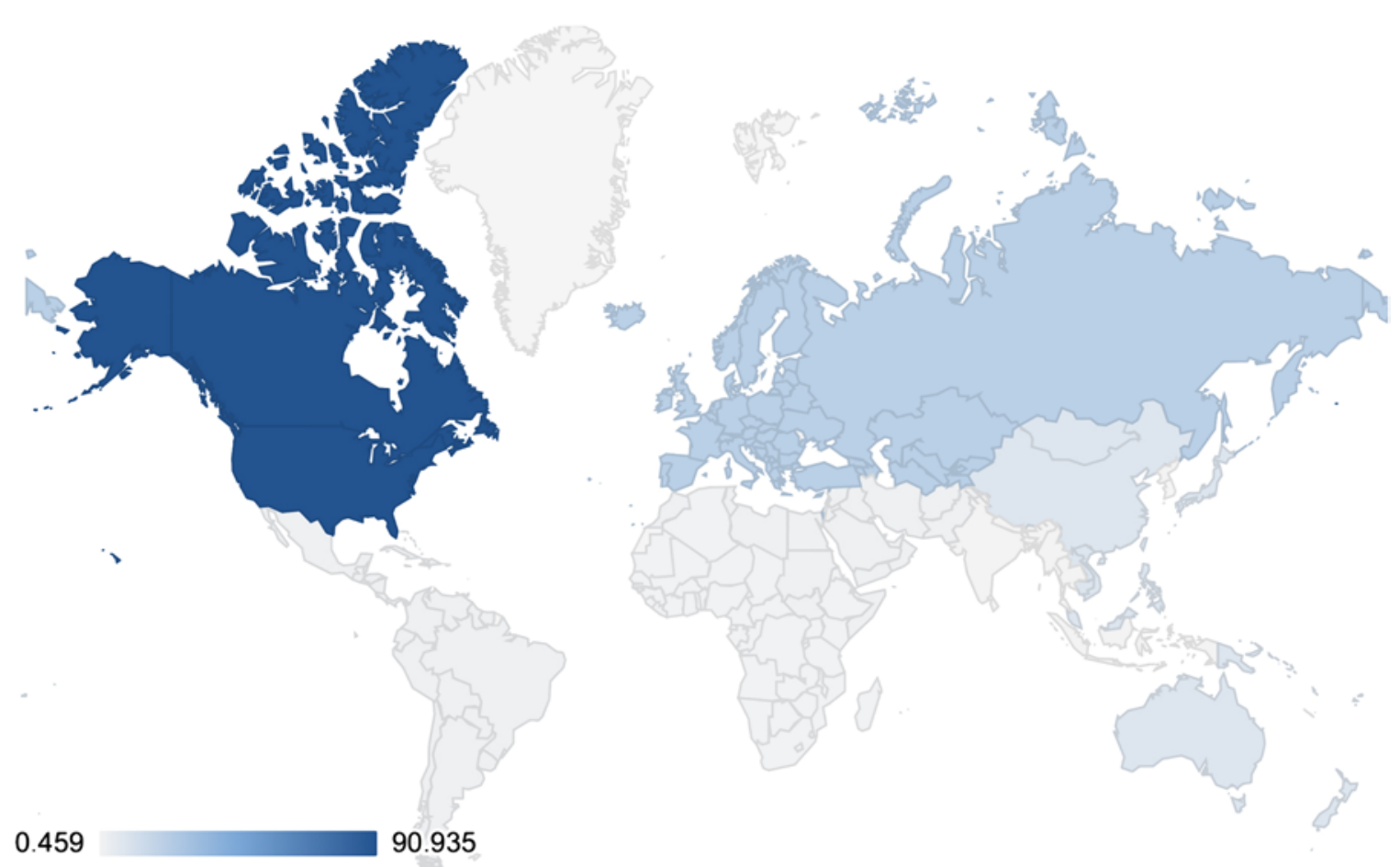

FIG. 3. Map showing the heterogeneity in the publication to TBI volume ratio across the globe. 
only less than $3 \%$ of all published papers. These regions have a population of 3.5 billion people, representing $46 \%$ of the total worldwide population. If we then examine single countries, the US, Canada, China, and Australia are the most productive countries, with an important increase in Chinese papers in the last few years. The second "line" was formed in the last 10 years by European countries (the United Kingdom, Germany, Italy, the Netherlands, France, and Sweden) with the addition of Japan and New Zealand. In 2018 many other countries entered into this group (Table 2) but India was the only one from the LMICs.

The relationship between the number of publications and the burden of TBI becomes even more clear using the publication to volume ratio, ranging from a ratio of 91 in the US and Canada, to a ratio of 22 in Europe, to a low of 1 in Africa and 0.5 in Southeast Asia (Table 3, Fig. 3). This trend is confirmed by recent data on randomized clinical trials (Hutchinson PJ, Kolias A, et al., unpublished data, 2019) where only 35 trials $(8.8 \%)$ were conducted in LMICs. As an example, all patients included in the decompressive "DECRA" study ${ }^{8}$ are from HICs as well as $91 \%$ of the patients included in the "RESCUEicp" (Randomised Evaluation of Surgery with Craniectomy for Uncontrollable Elevation of ICP) study. ${ }^{14}$ Furthermore, if we investigate the most cited papers in TBI, 67 of 100 are published in the US and the rest in Europe. ${ }^{16}$ The guidelines recently published on TBI management are the result of this disparity. ${ }^{4}$ Evidence-based medicine is produced by high-quality papers that are, in large part, originated from HICs where TBI is less than $30 \%$ of worldwide volume. Therefore, it is difficult to apply such guidelines in settings in which the resources, infrastructure, and staffing levels are completely different. ${ }^{26}$ There have been a series of appreciable suggestions from Western countries about how to address this issue. One example is the substitution of intracranial pressure (ICP) monitoring when it is not available with a series of CT scans to monitor for mass effect. ${ }^{3,6}$ Unfortunately, repeat CT is not possible because this would necessitate out-of-pocket expenses; therefore patient management must be based on this reality. ${ }^{1}$ This example shows how important it is to include papers from LMICs even if not scored as level of evidence class I or II, if we wish to produce worldwide applicable guidelines.

\section{What Can We Do to Reduce This Disparity?}

The production of suggestions on TBI management based on consensus conferences is a good way to include neurosurgeons from all over the world both in the discussion and in publications. ${ }^{13}$ Their views on indications are different from neurosurgeons in HICs and must be taken into account, such as the use of decompression as a "preventive" measure to avoid subsequent intracranial hypertension. The inclusion of some LMICs in large international databases of $\mathrm{TBI}^{17}$ is a good example of how to compare data from these areas with American and European patients. Another way is the establishment of simple prospective studies with a form and registries that can be electronically filled out all over the world (https:// globalneurotrauma.com). Some specialized selected centers from LMICs have been recently included in high-level clinical randomized trials. In the RESCUE-ASDH trial
(Randomised Evaluation of Surgery with Craniectomy for patients Undergoing Evacuation of Acute SubDural Haematoma) some sites from LMICs have actively participated and enrolled several patients (http://www.rescueasdh. org/home).

A recurring theme is the need to support the building of research capacity in LMICs. For most neurosurgeons working in the developing world, conducting research is a luxury. Research collaborations between surgeons from HICs and LMICs can lead to a sustainable increase in local research capacity but requires a long-term strategy and commitment as well as an ethical framework. Additionally, the root causes of the paucity of epidemiological data and papers ${ }^{21}$ from LMICs need to be addressed. Unless national surveillance and data collection capacity is strengthened, the true burden of TBI and other high-burden conditions will never be known or tracked. Here, the World Federation of Neurosurgical Societies can advocate through the WHO to include TBI data elements in national injury surveillance and trauma registries. One aspect of the publication process that has not been mentioned is the cost of publications and the paywall for accessing the articles. Solutions are needed that remove the financial barriers for authors from LMICs for publishing their research, as well as the economic possibility to access and read articles, for example, with free open access resources.

\section{Conclusions}

Our bibliometric analysis detected an important disparity that currently exists between countries with a high burden of TBI and those in which most of the research is conducted. Improving the care of patients with TBI on a worldwide perspective also means to better treat patients wherever they are. It will take substantial effort, time, and funds to conduct high-volume and quality sophisticated research originating from LMICs, but we need to encourage investigators from HICs to collaborate with colleagues from LMICs to improve their research capacity and guideline production.

\section{References}

1. Adeleye AO: Clinical and radiologic outcome of a less invasive, low-cost surgical technique of osteoplastic decompressive craniectomy. J Neurol Surg A Cent Eur Neurosurg 77:167-175, 2016

2. Al-Shahi R, Will RG, Warlow CP: Amount of research interest in rare and common neurological conditions: bibliometric study. BMJ 323:1461-1462, 2001

3. Alali AS, Temkin N, Barber J, Pridgeon J, Chaddock K, Dikmen S, et al: A clinical decision rule to predict intracranial hypertension in severe traumatic brain injury. J Neurosurg 131:612-619, 2019

4. Carney N, Totten AM: O'Reilly C, Ullman JS, Hawryluk GWJ, Bell MJ, et al: Guidelines for the management of severe traumatic brain injury, fourth edition. Neurosurgery 80:6-15, 2017

5. Cassidy JD, Carroll LJ, Peloso PM, Borg J, von Holst H, Holm L, et al: Incidence, risk factors and prevention of mild traumatic brain injury: results of the WHO Collaborating Centre Task Force on Mild Traumatic Brain Injury. J Rehabil Med 43 (43 Suppl):28-60, 2004

6. Chesnut RM, Temkin N, Dikmen S, Rondina C, Videtta W, 
Petroni G, et al: A method of managing severe traumatic brain injury in the absence of intracranial pressure monitoring: the imaging and clinical examination protocol. J Neurotrauma $35: 54-63,2018$

7. Cnossen MC, Lingsma HF, Tenovuo O, Maas AIR, Menon D, Steyerberg EW, et al: Rehabilitation after traumatic brain injury: a survey in 70 European neurotrauma centres participating in the CENTER-TBI study. J Rehabil Med 49:395401, 2017

8. Cooper DJ, Rosenfeld JV, Murray L, Arabi YM, Davies AR, D'Urso P, et al: Decompressive craniectomy in diffuse traumatic brain injury. N Engl J Med 364:1493-1502, 2011

9. Corley J, Lepard J, Barthélemy E, Ashby JL, Park KB: Essential neurosurgical workforce needed to address neurotrauma in low- and middle-income countries. World Neurosurg 123:295-299, 2019

10. Dewan MC, Rattani A, Gupta S, Baticulon RE, Hung YC, Punchak M, et al: Estimating the global incidence of traumatic brain injury. J Neurosurg 130:1080-1097, 2019

11. El-Menyar A, Mekkodathil A, Al-Thani H, Consunji R, Latifi $\mathrm{R}$ : Incidence, demographics, and outcome of traumatic brain injury in the Middle East: a systematic review. World Neurosurg 107:6-21, 2017

12. Hauptman JS, Chow DS, Martin NA, Itagaki MW: Research productivity in neurosurgery: trends in globalization, scientific focus, and funding. J Neurosurg 115:1262-1272, 2011

13. Hutchinson PJ, Kolias AG, Tajsic T, Adeleye A, Aklilu AT, Apriawan T, et al: Consensus statement from the International Consensus Meeting on the Role of Decompressive Craniectomy in the Management of Traumatic Brain Injury. Acta Neurochir (Wien) 161:1261-1274, 2019

14. Hutchinson PJ, Kolias AG, Timofeev IS, Corteen EA, Czosnyka M, Timothy J, et al: Trial of decompressive craniectomy for traumatic intracranial hypertension. N Engl J Med 375:1119-1130, 2016

15. Iaccarino C, Carretta A, Nicolosi F, Morselli C: Epidemiology of severe traumatic brain injury. J Neurosurg Sci 62:535-541, 2018

16. Li L, Ma X, Pandey S, Deng X, Chen S, Cui D, et al: The most-cited works in severe traumatic brain injury: a bibliometric analysis of the 100 most-cited articles. World Neurosurg 113:e82-e87, 2018

17. Maas AIR, Menon DK, Steyerberg EW, Citerio G, Lecky F, Manley GT, et al: Collaborative European NeuroTrauma Effectiveness Research in Traumatic Brain Injury (CENTERTBI): a prospective longitudinal observational study. Neurosurgery 76:67-80, 2015

18. Majdan M, Plancikova D, Brazinova A, Rusnak M, Nieboer $\mathrm{D}$, Feigin V, et al: Epidemiology of traumatic brain injuries in Europe: a cross-sectional analysis. Lancet Public Health 1:e76-e83, 2016

19. Nguyen R, Fiest KM, McChesney J, Kwon CS, Jette N, Frolkis AD, et al: The international incidence of traumatic brain injury: a systematic review and meta-analysis. Can J Neurol Sci 43:774-785, 2016

20. Peeters S, Blaine C, Vycheth I, Nang S, Vuthy D, Park KB: Epidemiology of traumatic brain injuries at a major government hospital in Cambodia. World Neurosurg 97:580-589, 2017
21. Roozenbeek B, Maas AIR, Menon DK: Changing patterns in the epidemiology of traumatic brain injury. Nat Rev Neurol 9:231-236, 2013

22. Rubiano AM, Carney N, Chesnut R, Puyana JC: Global neurotrauma research challenges and opportunities. Nature 527:S193-S197, 2015

23. Salhab HA, Salameh P, Hajj H, Hosseini H: Stroke in the Arab world: a bibliometric analysis of research activity (2002-2016). eNeurologicalSci 13:40-45, 2018

24. Servadei F, Rossini Z, Nicolosi F, Morselli C, Park KB: The role of neurosurgery in countries with limited facilities: facts and challenges. World Neurosurg 112:315-321, 2018

25. Servadei F, Tropeano MP, Spaggiari R, Cannizzaro D, Al Fauzi A, Bajamal AH, et al: Footprint of publications from low- and low- to middle-income countries in the neurosurgical data: a study from 2015 to 2017. World Neurosurg [epub ahead of print], 2019

26. Smart LR, Mangat HS, Issarow B, McClelland P, Mayaya G, Kanumba E, et al: Severe traumatic brain injury at a tertiary referral center in Tanzania: epidemiology and adherence to Brain Trauma Foundation guidelines. World Neurosurg 105:238-248, 2017

27. Taylor CA, Bell JM, Breiding MJ, Xu L: Traumatic brain injury-related emergency department visits, hospitalizations, and deaths - United States, 2007 and 2013. MMWR Surveill Summ 66:1-16, 2017

28. Tran TM, Fuller AT, Kiryabwire J, Mukasa J, Muhumuza M, Ssenyojo H, et al: Distribution and characteristics of severe traumatic brain injury at Mulago National Referral Hospital in Uganda. World Neurosurg 83:269-277, 2015

\section{Disclosures}

The authors report no conflict of interest concerning the materials or methods used in this study or the findings specified in this paper.

\section{Author Contributions}

Conception and design: Spaggiari, Tropeano, Ileyassoff. Acquisition of data: Spaggiari, Tropeano, Ileyassoff. Analysis and interpretation of data: Spaggiari, Tropeano, Ileyassoff. Drafting the article: Spaggiari, Tropeano, Ileyassoff, Kolias. Critically revising the article: Spaggiari, Park, Kolias, Hutchinson, Servadei. Reviewed submitted version of manuscript: Park, Kolias, Hutchinson, Servadei. Study supervision: Hutchinson, Servadei.

\section{Correspondence}

Riccardo Spaggiari: Humanitas Clinical and Research Hospital and Humanitas University, Rozzano, Italy. rickyspaggio945@ gmail.com. 